\title{
Acupuncture for patients with mild hypertension: study protocol of an open-label multicenter randomized controlled trial
}

\author{
Juan Li ${ }^{1}$, Hui Zheng ${ }^{1}$, Ling Zhao ${ }^{1}$, Ying $\mathrm{Li}^{1}$, Yan Zhang ${ }^{2}$, Xiao-rong Chang ${ }^{3}$, Rui-hui Wang ${ }^{4}$, Jing Shi ${ }^{5}$, Jin Cui ${ }^{6}$, \\ Yin-lan Huang ${ }^{7}$, Xiang Li ${ }^{1}$, Jie Chen ${ }^{1}$, De-hua Li ${ }^{1}$ and Fan-rong Liang ${ }^{1 *}$
}

\begin{abstract}
Background: Several studies using acupuncture to treat essential hypertension have been carried out. However, whether acupuncture is efficacious for hypertension is still controversial. Therefore, this trial aims to evaluate the efficacy and safety of acupuncture for patients with mild hypertension.

Methods/Design: This is a large scale, open-label, multicenter, randomized controlled clinical trial with four parallel arms. We will recruit 428 hypertensive patients with systolic blood pressure (SBP) between 140 and $159 \mathrm{mmHg}$, diastolic blood pressure (DBP) between 90 and $99 \mathrm{mmHg}$. The participants will be randomly assigned to four different groups (three acupuncture groups and one waiting list group) (1).The affected meridian acupuncture group $(n=107)$ is treated with acupoints on the affected meridians (2).The non-affected meridian acupuncture group $(n=107)$ is treated with acupoints on the non-affected meridians (3).The invasive sham acupuncture group $(n=107)$ is provided with sham acupoints treatment (4).The waiting-list group $(n=107)$ is not offered any intervention until they complete the trial. Each patient allocated to acupuncture groups will receive 18 sessions of acupuncture treatment over 6 weeks. This trial will be conducted in 11 hospitals in China. The primary endpoint is the change in average 24-hSBP before and 6 weeks after randomization. The secondary endpoints are average SBP and average DBP during the daytime and night-time, and 36-Item Short Form Survey (SF-36), and so on.
\end{abstract}

Discussion: This is the first large scale, multicenter, randomized, sham controlled trial of acupuncture for essential hypertension in China. It may clarify the efficacy of acupuncture as a treatment for mild hypertension.

Trials registration: Clinicaltrials.gov Identifier: NCT01701726.

Keywords: Essential hypertension, Acupuncture, Acupoint specificity, Acupoints effect, Randomized controlled trials, Protocol

\section{Background}

Essential hypertension continues to be an important public-health challenge worldwide [1]. Hypertension is an independent risk factor for premature death, heart disease, stroke, peripheral vascular disease, and renal failure. Hypertension affects $29.3 \%$ of the adult population in the United States [2]. In 2009, the direct and indirect costs for hypertension in the United States are projected to be approximately $\$ 73.4$ billion [3]. In the fourth National

\footnotetext{
* Correspondence: acuresearch@126.com

${ }^{1}$ Chengdu University of Traditional Chinese Medicine Chengdu, Chengdu, Sichuan, PR China

Full list of author information is available at the end of the article
}

Nutrition and Health Survey in 2002, the prevalence of hypertension among Chinese population was 18.8\% [4], affecting approximately 200 million individuals in China, while mild hypertension accounts for $>60 \%$ [5]. Furthermore, the number of hypertensive patients is still increasing [6]. It is also reported that in China, the direct medical costs for hypertensive patientsaged 35 to 75 years are $¥ 20.2$ billion [7].

Pharmacological treatment remains the chief treatment option for mild hypertension. However, a recent Cochrane review found that there is no proved benefit in antihypertensive drugs treatment for patients with mild hypertension. In addition, owing to adverse effects

\section{Biomed Central}

(c) 2013 Li et al.; licensee BioMed Central Ltd. This is an open access article distributed under the terms of the Creative Commons Attribution License (http://creativecommons.org/licenses/by/2.0), which permits unrestricted use, distribution, and reproduction in any medium, provided the original work is properly cited. 
of antihypertensive drugs, $9 \%$ of patients discontinue their treatment [8]. Non-pharmacological interventions including acupuncture prevail as a treatment option for hypertensive patients [9]. In China, acupuncture has been used to treat disease for $>2,500$ years, and it is also widely used all around the world. Nowadays, acupuncture is used to treat cardiovascular disease (such as angina pectoris [10], hypertension [11]). Several randomized controlled trials of acupuncture to treat hypertension had been conducted, but the results were not consistent. Therefore, we designed a multicenter randomized controlled trial to confirm whether acupuncture is efficacious and safe for hypertension.

In this trial, we first intend to investigate the efficacy of acupuncture to lower the blood pressure comparing the waiting-list control and invasive sham acupuncture groups. Second, we attempt to determine whether there is a difference between acupoints on the affected meridians according to meridian syndrome differentiation and the acupoints on the non-affected meridians. This clinical trial is financed by the national basic research program (973program) in China, and is registered with an identifier (NCT01701726) by clinicaltrials.gov in the USA.

\section{Methods/Design \\ Design}

This trial is an open-label multicenter randomized controlled trial with four parallel arms. We compare the affected meridians with non-affected meridians, sham acupuncture and waiting-list groups as well. The trial is performed in the following Hospitals: First Affiliated Hospital of Chengdu University of Traditional Chinese Medicine; Third Affiliated Hospital of Chengdu University of Traditional Chinese Medicine (TCM); Affiliated Hospital of Ningxia Medicine University; First Affiliated Hospital of Hunan University of TCM; Second Affiliated Hospital of Hunan University of TCM; Affiliated Hospital of Hunan University of TCM - Hengyang Hospital; Chenzhou No.1 People's Hospital; First Affiliated Hospital of Guiyang College of TCM; Second Affiliated Hospital of Guiyang College of TCM; Yunnan Provincial Hospital of TCM; and Second Affiliated Hospital of Shanxi College of TCM.

The whole study period is 13 weeks,with a 1-week run-in period, 6-week treatment phase, and 6-week follow-up phase. After randomization, patients in acupuncture groups will receive 18 sessions of treatment over a 6-week treatment period. Participants in the waiting-list control group will give an option to receive acupuncture treatment after 13 weeks study period. Outcome measurements will be assessed at day 0, 6 weeks, 9 weeks, and 12 weeks after randomization. All patients will be informed that they may be randomly allocated to affected meridian acupuncture group, non-affected acupuncture group, invasive sham acupuncture group, or waiting-list group (Figure 1, Table 1).
This trial will be conducted according to the principles of the Declaration of Helsinki (version Edinburgh 2000). The trial protocol has been approved by the regional ethical review committee of traditional Chinese medicine in Sichuan Province on April 2012. We will require all patients to sign the written informed consent, and they will be given enough time to decide whether they will take part in this trial. This trial started in September 2012, and will be completed in December 2017.

\section{Randomization}

Randomization is conducted by the third party (the Brightech Magnsoft Data Services Company) using text messages or a website. If the randomization succeeds, the investigators will receive an email confirmation. The randomization sequence (blocked, stratified for centers) is generated through the randomization module of the synthesized management platform of the Brightech Magnsoft Data Services Company (block length is unknown to centers). This procedure ensures each eligible patient having an equal probability of being assigned to four different groups, which will not be influenced by the researchers.

\section{Participants}

Patients who meet the inclusion criteria will be included in this study. The criteria are as follows: (1) men or women aged between 40 and 75 years,; (2) participants meet the diagnostic criteria of mild hypertension according to JNC-7 [12] and 2010 Chinese guidelines for the management of hypertension [5](diagnosed as mild hypertension systolic blood pressure (SBP) 140-159 and/or diastolic blood pressure (DBP) 90-99, according to WHO/ ISH criteria stage I); (3) patients who are initially diagnosed as mild hypertension or have been diagnosed as mild hypertension before, without taking any antihypertensive drugs; (4) patients who are diagnosed as Jue-yin syndrome (yin-deficiency accompany with hyperactivity of yang) or Yang-ming syndrome (obstruction of phlegm and dampness) according to meridian syndrome differentiation; (5) patients who are willing to comply with our study protocol; (6) participants agree to sign informed consent form.

The exclusion criteria are as follows: (1) patients who have been diagnosed with secondary hypertension [13] or malignant hypertension (for example, Cushing's syndrome, coarctation of the aorta, pheochromocytoma, renal parenchymal disease, primary aldosteronism, renovascular hypertension, obstructive sleep apnea, drug-induced hypertension, and so on); (2) patients accompanied with other severe medical conditions (for example, endocrine disorders, cardiovascular disease, digestive disease, hepatic dysfunction, cerebral vascular disease, renal disease, hematologic disease,and so on); (3) patients with a chronic disease (for example, epilepsy, severe depression, or anxiety (SAS $\geq 70$, 


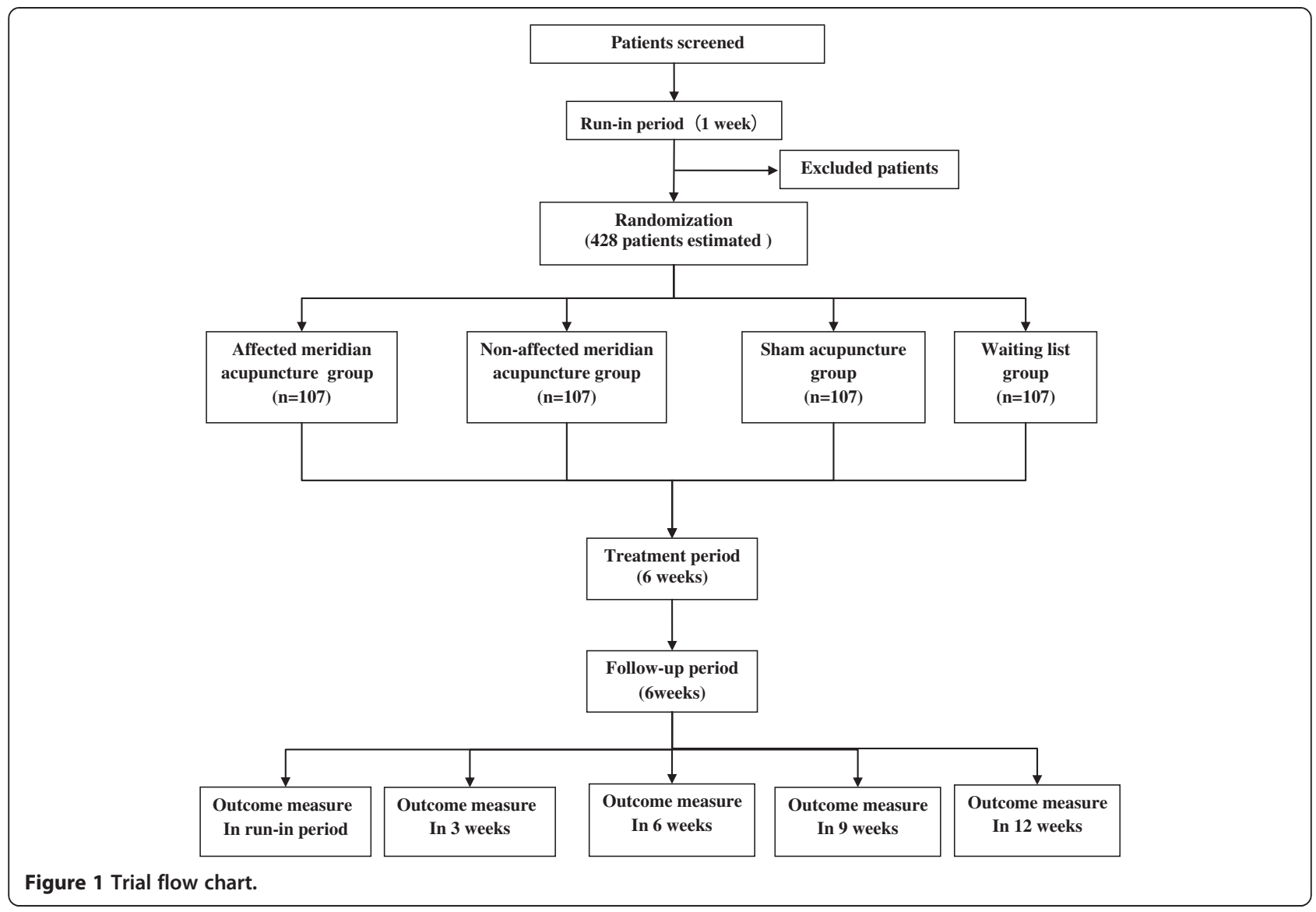

SDS $\geq 70$ ), psychosis, allergic constitution, accompany with any infection), pregnant women or women in lactation or women of childbearing potential planning to conceive in the next 6 months; (4) patients who currently participate in another clinical trial; (5) patients who had been treated with acupuncture during the previous 3 months. If one of the criteria mentioned above is matched, the participant will be excluded.

During the whole study period, participants will not be hospitalized, taking into account: (1) we only recruit patients with mild essential hypertension who went off hypertensive drugs and without complications; (2) we could observe the blood pressure of participants under regular activities; (3) patients with mild essential hypertension are not appropriate to recruit for inpatient treatment.

\section{Recruitment strategies}

We will apply two strategies to recruit participants with mild hypertension. First, we recruit participants from outpatient clinics of the participated hospitals. Second, we employ three kinds of advertisements to attract patients to join our study. We will distribute printed recruitment posters in the participated hospitals and the communities nearby. Meanwhile we will publish advertisement in local newspaper and broadcast television advertisements on the local channel.

\section{Intervention}

Rationale for selection of acupoints According to TCM theory, hypertension pertains to the coverage of vertigo and headache. Based on previous survey and expert consultation, Jue-yin syndrome (yin deficiency with yang hyperactivity) and Yang-ming syndrome (the obstruction of phlegm and dampness) are the most common syndromes in mild hypertension. Therefore, in accordance with meridian syndrome differentiation, we select acupoints on the Yang-ming, Jue-yin, and Shao-yin meridians. Hence, it is reasonable that the acupoints on the affected meridians would be most effective at treating mild hypertensive patients. Meanwhile we also choose acupoints on the non-affected meridians (Gallbladder, San-jiao, and Spleen meridians) which were reported to be useful to decrease blood pressure according to our previous literature research.

In our trial, there are four groups: (1) affected meridian group;(2) non-affected meridian group;(3) sham acupuncture group;and (4) waiting-list group (Table 2). Apart from the waiting-list group, the other patients 
Table 1 Time to visit and data collection

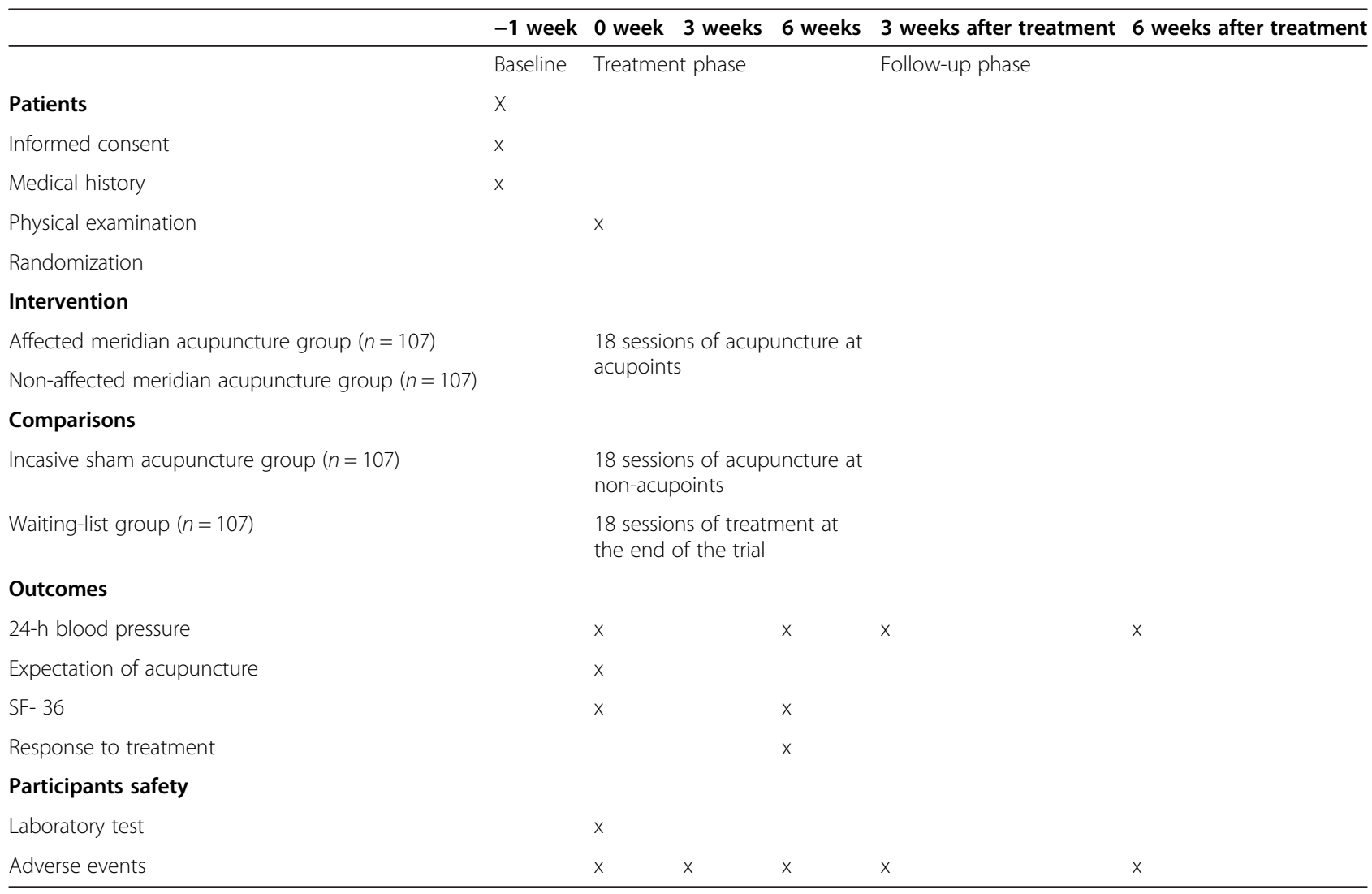

SAS, Self-Rating Anxiety Scale; SDS, Self-Rating Depression Scale; SF-36, MOS item Short Form health survey.

assigned to the three acupuncture groups will each receive 18 sessions of acupuncture treatment.

1. Affected meridian acupuncture (AMA) Participants in the affected meridian group (Figure 2) are treated according to two syndromes (Jue-yin syndrome and Yang-ming syndrome). Jue-yin syndrome: taichong (LR3), renying (ST9), taixi (KI3), and neiguan (PC6) are punctured with filiform needles bilaterally. Taichong (LR3) is punctured perpendicularly 0.5-1.0 cun. Renying (ST9) is punctured perpendicularly $0.3-0.8$ cun, paying attention to avoiding the carotid artery. Taixi (KI3) is punctured perpendicularly $0.5-1.5$ cun. Neiguan (PC6) is punctured perpendicularly 0.5-1.0 cun. Yang-ming syndrome: taichong (LR3), renying (ST9), zusanli (ST36), and quchi (LI11) are punctured with filiform needles bilaterally. Taichong (LR3) is punctured perpendicularly 0.5-1.0 cun. Renying (ST9) is punctured perpendicularly 0.3-0.8 cun, paying attention to avoiding the carotid artery. Zusanli (ST36) is punctured perpendicularly 1.0-2.0 cun. Quchi (LI11) is punctured perpendicularly 1.0-1.5 cun. After insertion, all the acupoints will be manually stimulated by lifting and thrusting associated with twirling and rotating the needle, to elicit 'de-qi'.

Table 2 Details of acupuncture protocol

\begin{tabular}{lll}
\hline AMA Jue-yin syndrome & Taichong (LR3), renying(ST9), taixi(KI3), neiguan(PC6) \\
YMan-ming syndrome & Taichong (LR3), renying(ST9), zusanli(ST36), quchi(LI11) \\
ISA & Fengchi(GB20), waiguan(SJ5), yinlingquan(SP9), xuehai(SP10) \\
& 1. The edge of the tibia (1-2 cm lateral and horizontal to the zusanli (ST36)) \\
& 3. On the ulnar side of the arm, halfway between the epicondylus medialis of the humerus and the ulnar side of the wrist \\
& 4. $2 \mathrm{~cm}$ superior to futu(LI18)
\end{tabular}




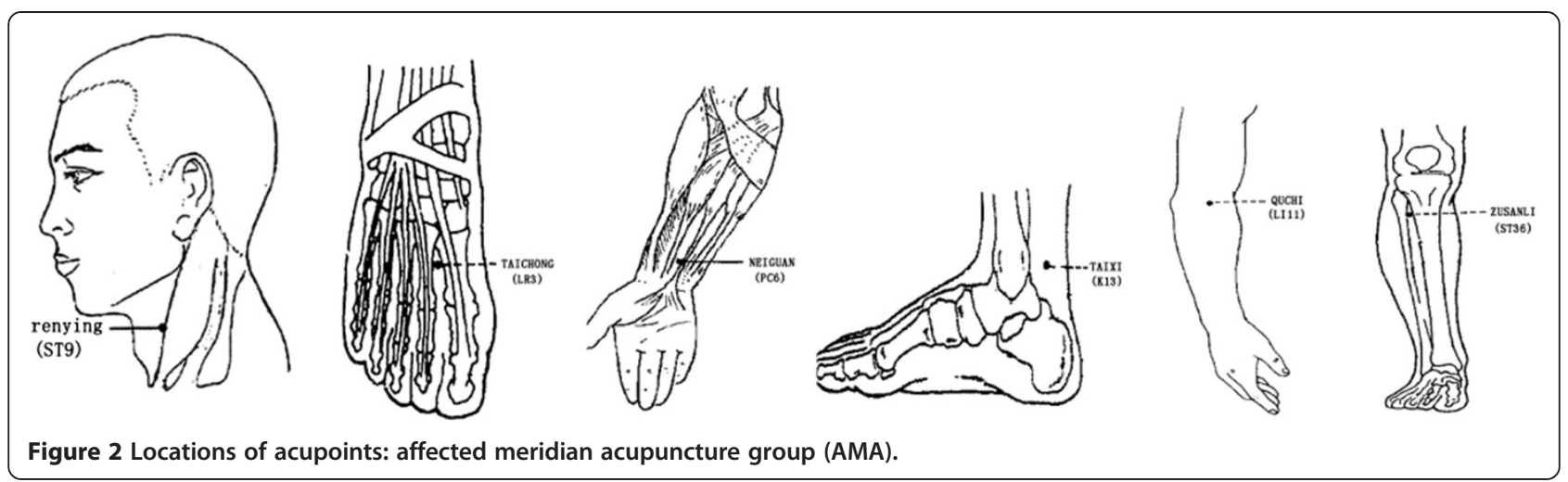

Jue-yin syndrome and Yang-ming syndrome will be identified by the practitioner according to the diagnostic characteristic variables of TCM (Table 3).

2. Non-affected meridian acupuncture (NMA) Fengchi (GB20), waiguan(SJ5), yinlingquan(SP9), and xuehai (SP10) are punctured by filiform needles bilaterally (Figure 3). Fengchi (GB20) is punctured obliquely 0.8-1.2cun, with the tip of the needle pointing to the apex of nose. Waiguan (SJ5) is punctured perpendicularly 0.5-1.0 cun. Yinlingquan (SP9) is punctured perpendicularly 1.0-2.0 cun. Xuehai (SP10) is punctured perpendicularly 1.0-1.5cun. All the acupoint locations are in accordance with the WHO Guideline for Acupuncture Point Locations [14]. After insertion, all the acupoints will be manually stimulated by lifting and thrusting associated with twirling and rotating the needle, to search for 'de-qi'.

3. Invasive sham acupuncture (ISA)

Sham acupoints (Figure 4):

(1) The edge of the tibia (1-2 cm lateral and horizontal to the zusanli (ST36))

(2)Halfway between the tip of the elbow and the axilla

(3) On the ulnar side of the arm, halfway between the epicondylus medialis of the humerus and the ulnar side of the wrist

(4) $2 \mathrm{~cm}$ superior to fu tu (LI18)

We use pre-validated sham acupoints [15]. After the insertion, we will not use any manipulationand 'de-qi' sensation is not needed.
4. Waiting-list (WL)

Participants in the WL group will not be treated throughout 13-week observation period. And they will be required to maintain their current lifestyle, including diet and exercise. At the end of trial, if the participant would like to be treated with acupuncture, we will offer acupuncture treatment three times weekly for 6 weeks.

During the whole study period, all the participants are not allowed to take any medical treatment for lowering blood pressure; drugs, herbs, and physical therapy are forbidden. If the participants suffer from other diseases and need to be treated with drugs,they have to record the drugs they take, and measure their blood pressure after drugs, all of which will be recorded in the case report forms.

In each session, acupuncture is applied bilaterally. After insertion, apart from the acupoints around the neck (ST9, GB20, non-acupoints4), six auxiliary needles will be punctured at $2 \mathrm{~mm}$ lateral to each acupoint or non-acupoint. As to the auxiliary needle, we will not do any manipulation. We use transcutaneous electric acupoints stimulation (HANS; Han's acupoints nerve stimulator, HANS-200, Nanjing, China) to stimulate the acupoints and nonacupoints. Except for the acupoints around the neck (ST9, GB20, non-acupoints 4), the other acupoints or nonacupoints and their auxiliary needles are connected with electricity by HANS for $30 \mathrm{~min}$, the frequency is $2 \mathrm{HZ}$, and

Table 3 Diagnostic characteristic variables of TCM

\begin{tabular}{|c|c|c|}
\hline & Yin deficiency with yang hyperactivity & Obstruction of phlegm and dampness \\
\hline Pulse & Wiry, thin,fast & Slippery and soggy \\
\hline Tongue & Red with no coating, peeled & Greasy coating, swollen \\
\hline $\begin{array}{l}\text { Other } \\
\text { primary } \\
\text { symptoms }\end{array}$ & $\begin{array}{l}\text { Vertigo, headache, malar flush, both knee and loin feel sore and weak, } \\
\text { dysphora in chest, palms, and soles, irritable, dry mouth, dry eyes, } \\
\text { palpitation, insomnia, poor memory }\end{array}$ & $\begin{array}{l}\text { Heavy head and or body, muddled thinking, } \\
\text { overweight, vertigo, palpitation, poor appetite, nausea, } \\
\text { stuffy feeling in the chest and/or epigastrum, heavy } \\
\text { frontal headache, insomnia }\end{array}$ \\
\hline $\begin{array}{l}\text { Secondary } \\
\text { symptoms }\end{array}$ & $\begin{array}{l}\text { Concentrated dark urine, dry stools, blurred vision, numbness and tremors of } \\
\text { limbs, tinnitus }\end{array}$ & Tinnitus, numbness in the limbs, loose stools \\
\hline
\end{tabular}



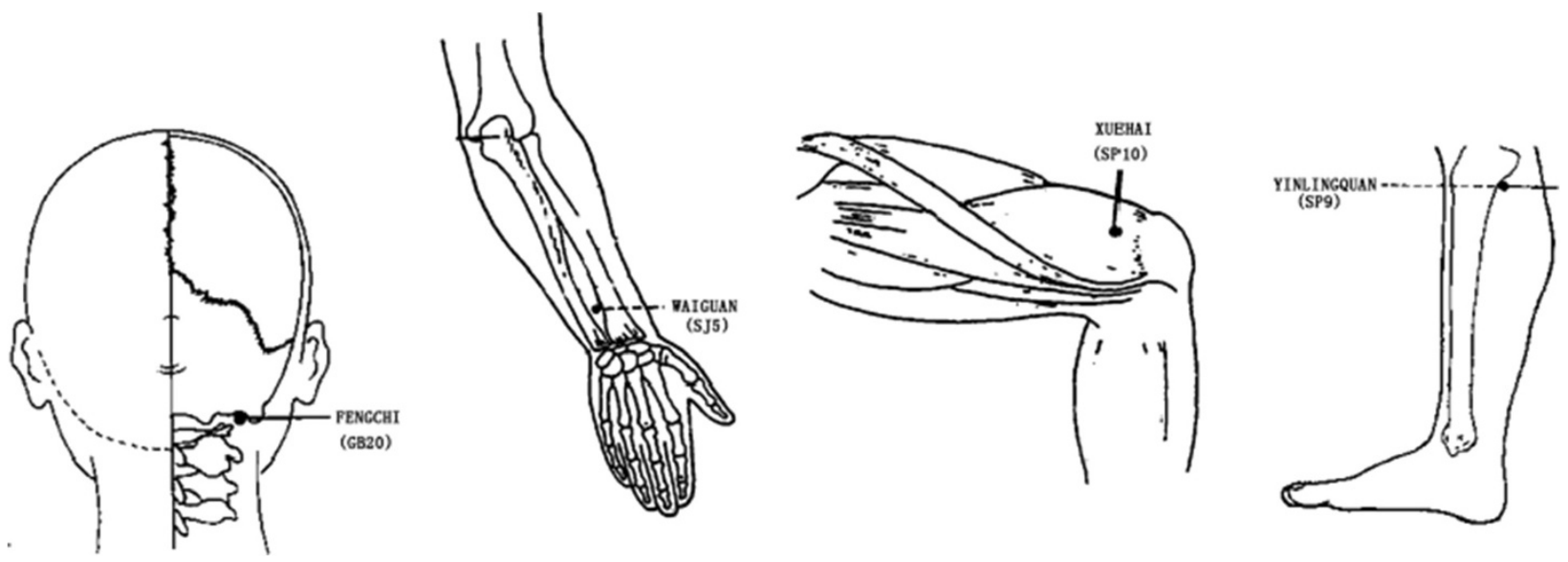

Figure 3 Locations of acupoints: non-affected meridian acupuncture group (NMA).

the intensity is confined to $2 \mathrm{~mA}$. It had been demonstrated that $30 \mathrm{~min}$ of low-frequency electro-acupuncture activates opioid receptors and provides a therapeutic effect on hypertension $[16,17]$.

The treatment will be performed after sterilizing the skin with $75 \%$ alcohol on the areas where the needles will be inserted, and with the patient lying in the supine position. We use two types of Hwato disposable steel needles (Suzhou Hua Tuo Medical Instuments, Suzhou, China): length 25-40 mm, diameter $0.25 \mathrm{~mm}$; and length $13 \mathrm{~mm}$, diameter $0.18 \mathrm{~mm}$. All the groups but the patients in waiting-list group are asked to retain the needles for $30 \mathrm{~min}$.

Apart from waiting-list group, the patients from the other groups will receive 18 sessions of acupuncture treatment over 6 weeks, three times per week. During the whole observation period, no patients are allowed to take antihypertensive drugs.

\section{Outcome measurement}

Primary endpoint The primary outcome measurement in this study is the change in average 24-hSBP and DBP before randomization and 6 weeks after randomization. Ambulatory mean blood pressure (ABPM) is superior to clinic BP in the prediction of cardiovascular morbidity and mortality $[18,19]$. Because ABPM exerts a different predictive effect on the cardiac and cerebrovascular complications [20] we selected ABPM as our primary endpoint.

Secondary outcome measures Ambulatory pulse pressure (APP) [21] is a well-established marker of cardiovascular risk. We choose APP as one of the secondary endpoints to evaluate the effect of acupuncture for hypertension. There is a prospective study [22] that shows that both high ABPM values and abnormal daytime/nighttime BP profiles are associated with later occurrence of

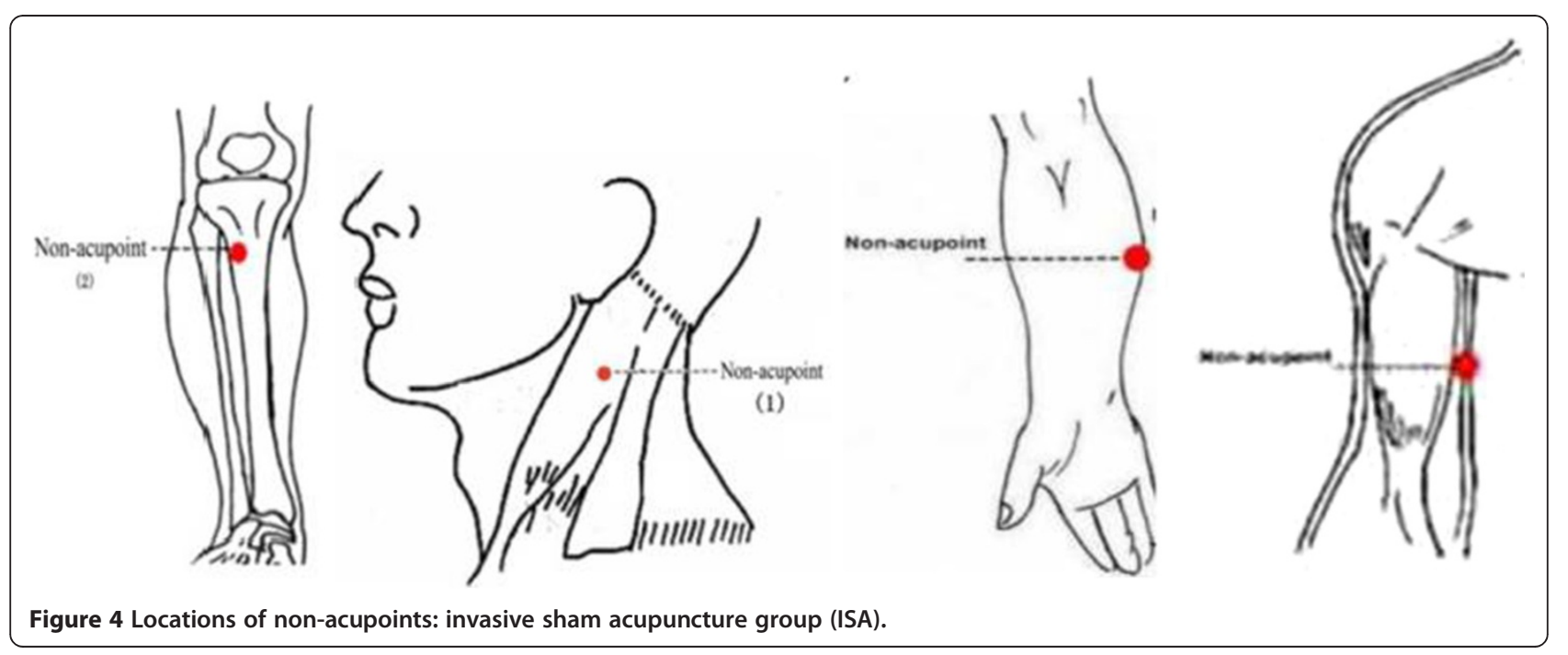


ischemic cerebrovascular and coronary events independently of casual BP values and other cardiovascular risk factors. It is therefore necessary to observe the daytime/ night-time BP of hypertensive patients.

Changes in patients' health-related quality of life, according to the SF-36 (Medical Outcomes Study 36-Item Short Form) questionnaire Chinese version is another secondary outcome measure [23]. The validity and reliability of this scale have proven in previous studies $[24,25]$. The scale contains eight dimensions (physical function, role physical, bodily pain, general health, vitality, social function, role emotional, mental health) and two summary components (physical and mental), measured on a scale of 0 to 100 . Lower scores indicate a poorer quality of life. SF-36 investigates at 0 day and 6 weeks after randomization.

Response to the treatment will be measured at the end of treatment, using a five-point Likert self-administered scale:'Compared with pre-treatment, to what extend did you think your hypertensive symptoms change?'1, absence of all symptoms; 2, significantly improved; 3, moderately improved; 4, not changed; 5, deteriorated. 'After treatment, how do you evaluate the effect of acupuncture for hypertension?' 1 , no effect( $0 \%)$; 2 , a little effect(25\%); 3, moderate effect(50\%); 4, a significant effect (75\%); 5, complete response(100\%). The patients fill in this questionnaire at the end of the treatment.

Expectation of the treatment will be measured by a self-administered questionnaire. With seven items as follows: (1) In the coming year, how will you think your disease will be relieved? ( $\operatorname{significantly}(\geq 75 \%)$; moderately (approximately $50 \%$ to $75 \%$ ); a little(approximately $25 \%$ to $50 \%$ ); no change( $0 \%$ to $25 \%)$ ); (2) How effective do you think acupuncture is for hypertension? (significant ( $\geq 75 \%$ ); moderate(approximately $50 \%$ to $75 \%$ ); a little (approximately 25\% to 50\%); no change(approximately $0 \%$ to 25\%)); (3) Which treatment options do you prefer to take as your treatment for hypertension? (acupuncture and moxibustion; herbs; Western medicine; others); (4) How familiar are you with acupuncture and moxibustion? (very familiar; so-so; a little; not at all); (5) By what means did you get to know about acupuncture and moxibustion? (friends and relatives; media reports; medical work; others); (6) How did acupuncture and moxibustion work in your memory? (excellent; good; so-so; not at all); (7) During the treatment, are you afraid of the pain or uncomfortable sensation caused by acupuncture and moxibustion? (very much; a little; never think about it; feel comfortable). The subjects will mark the best answer for each of the questions. The assessment will be performed at day 0 after randomization.

Before randomization, a special pattern identification questionnaire was developed to differentiate which syndrome the subjects pertaining to.

\section{Blood pressure measurement}

Ambulatory blood pressure measurements are performed with an oscillometric device (A\&D Co.Ltd., Japan TM2430). During a 24-h monitoring period, the device takes 76 measurements. Daytime is defined as 08:00-22:00. The measurement during the daytime will be taken every 15 min. Night-time is defined as 22:00-08:00. The measurement during the night-time will be taken every $30 \mathrm{~min}$. The 24-h ambulatory blood pressure monitoring measures at baseline, 6 weeks, 9 weeks, and 12 weeks after randomization. Clinical blood pressure measurements will be taken before and after each acupuncture session with Automatic Upper Arm Blood Pressure Monitor (Omron HEM 7200, Liaoning, China).

\section{Participant safety}

Before randomization, all participants will undergo routine tests of blood, urine, stool sample, electrocardiogram examination, Doppler echocardiography, carotid ultrasonography, as well as blood biochemical tests (AST, ALT, BUN, Scr, fasting glucose, triglyceride, HDL cholesterol, LDL cholesterol, albumin/creatinine.). These tests will help identify and exclude participants with serious illnesses of the heart, liver, and kidneys, or other severe diseases.

Adverse events are defined as any unexpected or uncomfortable signs, symptoms, or diseases, regardless of the intervention. These adverse events include bleeding, hematoma, fainting, serious pain, and local infection. If any adverse events happen during the entire observation period, all the details should be documented in the case report form.

We have designed a case reported form containing a variety of information, and this will be completed by the corresponding researchers at each center. At each center, the obtained information will be recorded on an electronic database, for subsequent statistical analysis. During the observation period, if there are any drop-outs or withdrawals, the investigator must find out their reasons and record them in the case report form. The patients' compliance during the 6 weeks of treatment and 6 weeks of follow-up will also be documented.

\section{Sample size calculation and statistical analysis}

According to the previous literature [26], the average 24-hSBP of hypertensive patients rose $1.6 \mathrm{mmHg}$ after being treated by sham acupuncture, while the average 24-hSBP of hypertensive patients lowered $5.4 \mathrm{mmHg}$ after being treated with verum acupuncture. Therefore, the adjusted mean difference between the two groups was $7 \mathrm{mmHg}$. In this study, we anticipated an $12 \mathrm{mmHg}$ improvement by affected meridian acupuncture; 10 $\mathrm{mmHg}$ by non-affected meridian acupuncture; $3 \mathrm{mmHg}$ by sham acupuncture; and $0 \mathrm{mmHg}$ in the waiting-list 
group. The standard deviation is set as 20 . We calculated this using $90 \%$ power at a $5 \%$ significance level with G*Power (version 3.1.2, Franz Faul, Universität Kiel, Germany), and the result showed an estimated 93 patients per group. Considering a drop-out rate of $15 \%$, we plan to enroll 107 patients per group, 428 patients in total for the four different groups.

Statistical analysis protocol was designed by the Brightech-Magnsoft Data Services Company and principal investigators. Analysis of all data in this trial will be performed by the Brightech-Magnsoft Data Services Company. The data will be analyzed with SAS 9.1 and SPSS13.0 software packages. All analyses will be on the basis of the intention-to-treat (ITT) population and perprotocol (PP) population. The result of the ITT analysis will be compared with that of the PP analysis to determine whether the results are consistent. Missing data will be filled by Last Observation Carry Forward rules.

Demographic characteristics and other baseline values will be described using descriptive statistics for each group. For the primary outcome, we will run a comparison between pooled acupuncture groups and waiting list control group to figure out whether acupuncture is more effective for this condition. We will then run another comparison between the pooled acupuncture groups and sham acupuncture group to find out whether acupuncture is more efficacious. Finally, a comparison between acupuncture groups will be run to see if selecting points along meridians was a better treatment option. The above analysis will be performed using the ANCOVA model. Multiple comparisons will be adjusted according to the Bonferroni correction method.

The repeated measures analysis will be used in the different time point assessments for the secondary outcomes. The Kruskal-Wallis test will be employed in the analysis of skewed distribution data. There is a designated acupuncturist in each center to all the acupuncture treatment, which probably introduces clustering effect in this trial. Therefore, we calculated the intra-cluster correlation coefficient from the results of this trial and report the coefficient.

\section{Discussion}

Acupoints are the regions where qi and blood from the viscera and meridians effuse and infuse beneath the body surface; the meridians and collaterals internally pertain to viscera and externally connect with the extremities. Therefore, stimulating the acupoints on the affected meridians can produce specific effects on regulating the corresponding organs, and the specificity of acupoint effect is the important basis for this regulation effect $[27,28]$. However, the specificity of acupoint effect on the affected meridians, continues to be a conflicting theoratical issue in acupuncture field [29,30]. In recent years, much research on acupoint specificity has been launched, but the resultsare still inconclusive. There is a small sample study of 41 randomized hypertensive patients into two groups (one gave verum acupuncture, and the other received non-penetrating sham acupuncture). After 17 treatment sessions, the verum acupuncture group showed a significant decrease in blood pressure [31]. Similar results had been found in another study, which discovered that unlike sham acupuncture, after 6 weeks of treatment with acupuncture according to TCM, the verum acupuncture significantly decreased mean 24-h ambulatory blood pressure [26]. On the other hand, another study of a higher quality obtained negative results on comparing active acupuncture with invasive sham acupuncture. They founded that active acupuncture provided no greater benefit than invasive sham acupuncture in reducing SBP or DBP [32].

Therefore, we launch this trial by means of multiple comparisons of four different arms (affected meridian acupuncture, non-affected meridian acupuncture, invasive sham acupuncture, and waiting-list groups) to aim to clarify this issue. First, we compare the efficacy of acupoints on the affected meridian with the waiting-list group who do not receive any intervention during the whole observation period. Second, we compare this with the invasive sham acupuncture group. We then compare the acupoints on the non-affected meridians. We believe that affected meridians based on syndrome differentiation acupuncture are capable of lowering the elevated blood pressure of hypertensive patients, of which effect is better than non-affected meridian acupuncture, and to a greater degree than the sham acupuncture and waiting-list groups. It can enhance health-related quality of life. Furthermore, as a treatment option for hypertension, it caused less adverse side effects. Through this trial, we attempt to demonstrate the real effectiveness of acupuncture for hypertension, and illustrate the specific effect of acupoints on the affected meridian.

Design highlights: first, unlike previous trials, we only include mild hypertensive patients without serious complications as it is safe for all subjects to go off hypertensive drugs and only be treated with acupuncture. We also rule out the influence from hypertensive drugs. Besides, by focusing on mild hypertension [33], we can cut down the compounding effect from different severities of hypertension. Second, we use electro-stimulation in the acupuncture groups aiming to ensure the stimulations between groups and individualsare equal. Third, in order to ensure the quality of this trial, we require that all the participating acupuncturists have at least 5 years of training and 5 years of practical experience. Before the trial, they will take a training course which includes the study protocol, methods for acupuncture treatment, and operating demonstration for this trial. After the 
course, they need to take an exam about the training course to be qualified for this trial. Meanwhile, we will designate special clinical monitors to check the progress of the trial and audit the accuracy and validity of the original data at each participating hospital once a month. Fourth, in our trial, a first ever large sample of patients will be recruited, which will make the results more powerful and convincing. Moreover, our design has a good understanding of theory of TCM and acupuncture.

One important limitation that may be present in this study is that it is an open-label trial. We design a waiting-list group as a blank controlled group. It is impossible to execute a blinding method. In order to overcome this problem, the acupuncturist and the outcome assessors are different, with respect to making the outcome assessment objectively. We also prevent the investigators and statisticians from randomization, to make sure all of them are blind to allocation. Among the three acupuncture groups, we conceal the patients from assignation so the patients do not know which acupuncture group they are allocated in.

This is a large scale, multicenter, randomized, controlled trial, and utilizes high quality methodologies. It may provide evidence for the effectiveness of acupuncture as a treatment for mild hypertension and confirm the existence of special effect of acupoints on affected meridians based on meridian syndrome differentiation.

\section{Trial status}

The first participant was included on 30 September 2012, and this article was submitted on 30 June. To date, 184 participants have been recruited.

\section{Competing interests}

The authors declare that they have no competing interests.

\section{Authors' contributions}

$J L, H Z, Y L, L Z, D H L, X L, J C, Z Y$, and $F R L$ participated in the conception and design of the trial. Planning the analysis of the data and drafting the manuscript. XRC RHW, JC, JS, and YLH participated in data collection and in charge of recruitment and treatment of patients in each center. All authors read and approved the final manuscript.

\section{Acknowledgements}

We gratefully acknowledge the Brightech-Magnsoft Data Services Company, for their cooperation and technological support about the central randomization, the protocol of statistical analysis, and quality supervision of this study. We would like to express our gratitude to the Huai-bin Du, Jian Zhang, and Zheng Yu from the acupuncture department of Third Affiliated Hospital of Chengdu University of Traditional Chinese Medicine, for their cooperation and material support. We also appreciate the hypertensive patients taking part in this trial. This trial was supported financially by the National Basic Research Program of China '973Program' (No. 2012CB518501).

\section{Author details}

${ }^{1}$ Chengdu University of Traditional Chinese Medicine Chengdu, Chengdu, Sichuan, PR China. ${ }^{2}$ First Affiliated Hospital, Chengdu University of Traditional Chinese Medicine, Chengdu, Sichuan, PR China. ${ }^{3}$ Hunan University of Traditional Chinese Medicine, Changsha, Hunan, PR China. ${ }^{4}$ Shanxi College of Traditional Chinese Medicine, Xi'an, Shanxi, PR China. ${ }^{5}$ Yunnan Provincial Hospital of Traditional Chinese Medicine, Kunming, Yunnan, PR China.
${ }^{6}$ Guiyang College of Traditional Chinese Medicine, Guiyang, Guizhou, PR China. ${ }^{7}$ Ningxia Medical University, Ningxia, Gansu, PR China.

Received: 30 June 2013 Accepted: 28 October 2013

Published: 11 November 2013

\section{References}

1. Kearney PM, Whelton M, Reynolds K, Muntner P, Whelton PK, He J: Global burden of hypertension: analysis of worldwide data. Lancet 2005, 365:217-223.

2. Ong KL, Cheung BM, Man YB, Lau CP, Lam KS: Prevalence, awareness, treatment, and control of hypertension among United States adults 1999-2004. Hypertension 2007, 49:69-75.

3. Lloyd-Jones D, Adams R, Carnethon M, De Simone G, Ferguson TB, Flegal K, Ford E, Furie K, Go A, Greenlund K: Heart disease and stroke statistics-2009 update a report from the American heart association statistics committee and stroke statistics subcommittee. Circulation 2009, 119:480-486.

4. Li LM, Rao KQ, Kong LZ, Yao CH, Xiang HD, Zhai FY, Ma GS, Yang XG: A description on the Chinese national nutrition and health survey in 2002. Chin J Epidemiol 2005, 26:478-484.

5. Liu LS: Chinese guidelines for management of hypertension. Chin J Hypertens 2010, 2011(19):701-708.

6. Wang J-G, Li Y: Characteristics of hypertension in the Chinese population. Curr Hypertens Rep 2012, 14:410-415.

7. Zhai Y, HU JP, Kong LZ, Zhao WH, Chen CM: Economic burden of coronary heart disease and stroke attributable to hypertension in China. Chin J Epidemiol 2006, 27:744-747.

8. Diao D, Wright JM, Cundiff DK, Gueyffier F: Pharmacotherapy for mild hypertension. Cochrane Database Syst Rev 2012, 8:CD006742.

9. Lin MC, Nahin R, Gershwin ME, Longhurst JC, Wu KK: State of complementary and alternative medicine in cardiovascular, lung, and blood research: executive summary of a workshop. Circulation 2001 , 103:2038-2041.

10. Richter A, Herlitz J, Hjalmarson A: Effect of acupuncture in patients with angina pectoris. Eur Heart J 1991, 12:175-178.

11. Zhou W, Longhurst JC: Neuroendocrine mechanisms of acupuncture in the treatment of hypertension. Evid Based Complement Altern Med 2011, 2012:878673.

12. Chobanian AV, Bakris GL, Black HR, Cushman WC, Green LA, Izzo JL, Jones DW, Materson BJ, Oparil S, Wright JT: Seventh report of the joint national committee on prevention, detection, evaluation, and treatment of high blood pressure. Hypertension 2003, 42:1206-1252.

13. Mancia G, De Backer G, Dominiczak A, Cifkova R, Fagard R, Germano G, Grassi G, Heagerty AM, Kjeldsen SE, Laurent S: Guidelines for the management of arterial hypertension the task force for the management of arterial hypertension of the European society of hypertension (ESH) and of the European society of cardiology (ESC). Eur Heart J 2007, 2007(28):1462-1536.

14. WHO Regional Office for the Western Pacific: WHO standard acupuncture point locations in the western pacific region. Manila: WHO; 2008.

15. Li Y, Zheng H, Witt CM, Roll S, Yu S-g, Yan J, Sun G-j, Zhao L, Huang W-j, Chang $X$ : Acupuncture for migraine prophylaxis: a randomized controlled trial. Can Med Assoc J 2012, 184:401-410.

16. Zhou W, Fu L-W, Tjen-A-Looi SC, Li P, Longhurst JC: Afferent mechanisms underlying stimulation modality-related modulation of acupuncturerelated cardiovascular responses. J App/ Physio/ 2005, 98:872-880.

17. Zhou W, Tjen-A-Looi SC, Longhurst JC: Brain stem mechanisms underlying acupuncture modality-related modulation of cardiovascular responses in rats. J Appl Physiol 2005, 99:851-860.

18. Health Quality Ontario: Twenty-four-hour ambulatory blood pressure monitoring in hypertension: an evidence-based analysis. Ont Health Technol Assess Ser 2012, 12:1-65.

19. Clement DL, De Buyzere ML, De Bacquer DA, de Leeuw PW, Duprez DA, Fagard RH, Gheeraert PJ, Missault LH, Braun JJ, Six RO: Prognostic value of ambulatory blood-pressure recordings in patients with treated hypertension. N Engl J Med 2003, 348:2407-2415.

20. Verdecchia P, Schillaci G, Reboldi G, Franklin SS, Porcellati C: Different prognostic impact of 24-hour mean blood pressure and pulse pressure on stroke and coronary artery disease in essential hypertension. Circulation 2001, 103:2579-2584. 
21. Verdecchia P, Schillaci G, Borgioni C, Ciucci A, Pede S, Porcellati C: Ambulatory pulse pressure a potent predictor of total cardiovascular risk in hypertension. Hypertension 1998, 32:983-988.

22. Bastos J, Bertoquini S, Silva J, Polonia J: Relationship between ambulatory blood pressure monitoring values and future occurrence of ischemic cerebrovascular and coronary events in hypertensive patients. Rev Port Cardiol 2006, 25:305.

23. Fang JQ: Quality of life measurement method and application. Peking University Medical Press; 2000.

24. Wang $C H$, Fang JQ, Tang XL, Zhang CZ, Lu YB, Meng Q, Gao L: The effect evaluation on measuring quality of life in patients with liver cancer by SF-36 scale. Tumor 2005, 25:492-494.

25. Wang $\mathrm{CH}$, Fang JQ, Shi ML, Chen LY, Zhang YZ: A comparative study on measuring quality of life for drug addicts by SF-36 scale. Chin J Behav Med Sci 1998, 7:260-261.

26. Flachskampf FA, Gallasch J, Gefeller O, Gan J, Mao J, Pfahlberg AB, Wortmann A, Klinghammer L, Pflederer W, Daniel WG: Randomized trial of acupuncture to lower blood pressure. Circulation 2007, 115:3121-3129.

27. Liang $F$, Fang $Z$, Zhao L, Tang Y: Specificity of acupoint effects and its fundamental laws. Zhongguo Zhen Jiu 2009, 29:129.

28. Ding $X Y$, She YF, Ma LX, Zhu J: Three basic characteristics of specificity of acupoint effect. China J TradiChin Medic Pharma 2010, 25:1356-1359.

29. Choi EM, Jiang F, Longhurst JC: Point specificity in acupuncture. Chin Med 2012, 7:1-5.

30. Zhao L, Chen J, Liu C-Z, Li Y, Cai D-J, Tang Y, Yang J, Liang F-R: A review of acupoint specificity research in china: status quo and prospects. Evid Based Complement Alter Medic 2012, 2012:543943.

31. Yin C, Seo B, Park H-J, Cho M, Jung W, Choue R, Kim C, Park H-K, Lee H, Koh H: Acupuncture, a promising adjunctive therapy for essential hypertension: a double-blind, randomized, controlled trial. Neurol Res 2007, Suppl 1:98-103.

32. Kalish LA, Buczynski B, Connell P, Gemmel A, Goertz C, Macklin EA, Pian-Smith M, Stevens S, Thompson J, Valaskatgis P: Stop hypertension with the acupuncture research program (SHARP): clinical trial design and screening results. Control Clin Trials 2004, 25:76-103.

33. The National Heart, Lung, and Blood Institute Working Group on Future Directions in Hypertension Treatment Trials: Major clinical trials of hypertension: what should be done next? Hypertension 2005, 46:1-6.

doi:10.1186/1745-6215-14-380

Cite this article as: Li et al: Acupuncture for patients with mild hypertension: study protocol of an open-label multicenter randomized controlled trial. Trials 2013 14:380.

\section{Submit your next manuscript to BioMed Central and take full advantage of:}

- Convenient online submission

- Thorough peer review

- No space constraints or color figure charges

- Immediate publication on acceptance

- Inclusion in PubMed, CAS, Scopus and Google Scholar

- Research which is freely available for redistribution 